\title{
Des lasers à excimères pour cristalliser le silicium des écrans plats : pourquoi ? comment?
}

\author{
C. Prat
}

New Display Technologies Laboratory, Thomson Multimedia R\&D France, 1 avenue Belle Fontaine, 35511 Cesson Sevigne, France

\begin{abstract}
Résumé. Les contraintes techniques et économiques de la fabrication d'écrans plats à cristaux liquides à matrice active ont suscité de nombreuses études de procédés de cristallisation de couches minces de silicium par laser à excimères, exploitant principalement trois types de phénomènes physiques, dans le but d'obtenir des cristaux micrométriques les plus uniformes possible.
\end{abstract}

\section{ECRANS A MATRICE PASSIVE ET ECRANS A MATRICE ACTIVE}

Une des caractéristiques (par rapport à un écran à tube cathodique) d'un écran plat à cristaux liquides ou à OLED (Organic light Emitting Diode) est l'organisation de sa surface d'affichage en pixels dont l'état allumé ou éteint constitue l'image. Le pixel est, dans le cas le plus simple de la matrice passive, défini par la surface d'intersection des électrodes linéaires disposées de part et d'autre du cristal liquide ou du matériau émissif (dans le cas des OLED par exemple) et orientées orthogonalement les unes par rapport aux autres.

Dans la méthode d'adressage adoptée pour les écrans matriciels chaque ligne est sélectionnée pendant $\mathrm{T} / \mathrm{N}$ ( $\mathrm{T}$ : période image; $\mathrm{N}$ : nombre de lignes de l'écran), durée pendant laquelle les pixels appartenant à la ligne considérée sont allumés (selon les besoins de l'image). Dans le cas de transducteurs sans mémoire ni rémanence, il est nécessaire de 'rafraîchir' l'image à une fréquence égale ou supérieure à $50 \mathrm{~Hz}$ pour éviter le papillotement. La période image T est doit donc être inférieure ou égale a $20 \mathrm{~ms}$. Les électrodes d'un pixel donné étant partagées avec tous les autres pixels de la même ligne et de la même colonne, la valeur de la tension sur ce pixel n'est maîtrisée que pendant la durée de sélection de la ligne, celui-ci subissant pendant tout le reste de la période image des tensions parasites. Les transducteurs dits "lents" (comme ceux utilisant des cristaux liquides nématiques) dont le temps de réponse est de l'ordre de grandeur d'une période image sont particulièrement sensibles à ces tensions parasites d'autant plus nuisibles au contraste de l'écran que celui comporte un grand nombre de lignes. On démontre que le contraste de l'écran devient inacceptable quand le nombre de lignes est supérieure à 100 ou 200, selon le type de cristal liquide utilisé [1].

Dans le cas de transducteurs rapides, comme les OLED, le problème majeur consiste à fournir au pixel l'énergie électrique suffisante pour produire l'effet lumineux souhaité pendant un temps T/N. Les courants nécessaires dépendent directement de la définition de l'écran : pour conserver un même niveau d'émission lumineuse, il faut augmenter les valeurs des courants à fournir proportionnellement au nombre de lignes. Cette augmentation s'accompagne d'un accroissement des pertes d'énergie dans les colonnes de l'écran et peut, dans le cas des écrans à OLED, endommager le matériau constitutif du pixel ou du moins, limiter considérablement sa durée de vie.

La solution retenue pour contourner ces limitations du multiplexage d'une matrice passive est la matrice active: chaque pixel est alors défini par une électrode qui lui est propre et commandé individuellement par un composant électronique. Ce composant est souvent associé à une capacité permettant de conserver l'information pendant une durée supérieure au temps de sélection d'une ligne. Le composant le plus répandu aujourd'hui pour ce type d'écran est le transistor à film mince (ou Thin Film Transistor: TFT). La difficulté accrue de la fabrication inhérente à cette technologie est largement justifiée par la demande d'écrans de grande dimension ou à haute résolution. 


\section{LA FABRICATION DES TFT}

L'élément clef de la fabrication de la matrice de TFT est la couche de silicium amorphe de 40 à $80 \mathrm{~nm}$ d'épaisseur, déposée sur un substrat de verre. Les TFT en silicium amorphe (a-Si) se caractérisent, entre autre, par une mobilité d'effet de champ n'excédant pas $1 \mathrm{~cm}^{2} / \mathrm{V}$.s. (TFT de type $n$ ) mais suffisante pour commander les pixel. Cependant, la nécessité d'intégrer à la périphérie des écrans tout ou partie des fonctionnalités de leurs drivers (onéreux à fabriquer séparément et à connecter sur l'écran), a poussé les fabricants à améliorer les performances du silicium amorphe en le cristallisant afin d'obtenir des mobilités de transistor de 70 à $100 \mathrm{~cm}^{2} / \mathrm{V}$.s indispensables à cette intégration. L'une des méthodes de cristallisation les plus usitées dans l'industrie des LCD (Liquid Crystal Display) est le traitement par laser à excimères $\mathrm{XeCl}$ révélé au milieu des années 80 [2]. Du fait de l'absorptivité du silicium à $308 \mathrm{~nm}$ et de la brièveté des impulsions laser, cette technique présente l'avantage de permettre la cristallisation tout en évitant que le substrat ne soit soumis à de trop fortes contraintes thermiques : ce substrat peut donc être en verre et bon marché. Cette technique est dite Low Temperature Poly Silicon (LTPS).

\subsection{Technologies laser disponibles}

Les deux types de sources laser aujourd'hui commercialement disponibles pour le recuit de silicium n'ont en commun que la nature de leur milieu actif basé sur la molécule $\mathrm{XeCl}$. La première développée par la société Lambda Physics (Allemagne) délivre une énergie maximale de $1 \mathrm{~J}$ (en sortie laser) en une impulsion de $35 \mathrm{~ns}$ environ à une fréquence de $300 \mathrm{~Hz}$. Le faisceau est projeté sur le silicium à cristalliser sous forme d'une ligne d'environ $400 \mu \mathrm{m}$ de large sur 200 à $350 \mathrm{~mm}$ de long. Le traitement de la moindre surface nécessite un balayage par des impacts d'autant plus nombreux qu'ils se recouvrent à 90 ou $95 \%$ de leur largeur pour assurer une bonne uniformité du recuit. Ce système (laser et homogéneiseur) est concurrencé par celui de la société SOPRA (France) dont la source laser produit $20 \mathrm{~J}$ en une impulsion de $200 \mathrm{~ns}$ à une fréquence de $1 \mathrm{~Hz}$. L'énergie délivrée par chaque impulsion autorise le traitement de surfaces l'ordre de $20 \mathrm{~cm}^{2}$ sans avoir recours au balayage.

\subsection{Les régimes de cristallisation}

L'irradiation d'un film de silicium amorphe donne lieu à trois régimes de cristallisation distincts en fonction de la densité d 'énergie utilisée.

- régime à faible densité d'énergie :

A de faibles densités d'énergie, l'absorption du rayonnement sur une épaisseur d'environ $10 \mathrm{~nm}$ provoque la fusion du silicium qui se cristallise instantanément en libérant une énergie suffisante pour amener à fusion la couche sous-jacente. Ce front de fusion-cristallisation se déplace à des vitesses comprises entre 10 et $20 \mathrm{~m} / \mathrm{s}$ et laisse au-dessus de lui un matériau cristallisé dont le diamètre moyen des grains est très inférieure à l'épaisseur de la couche de silicium. Durant cette phase, l'impulsion laser n'étant pas terminée, l'énergie laser continue à s'accumuler en surface entretenant un nouveau front de fusion qui se déplace à la vitesse de 4 à $6 \mathrm{~m} / \mathrm{s}$. Lors de la solidification, les microcristaux précédemment crées servent de germes à la formation de nouveaux grains dont la taille croît avec la densité d'énergie (et l'épaisseur de silicium fondu) $[3,4,5]$.

- régime de 'Super Lateral Growth' (SLG). [6,7].

En augmentant la densité d'énergie incidente, la couche de Si est entièrement fondue à l'exception de quelques microcristaux à partir desquels les grains prennent naissance des grains dont le diamètre peut atteindre 10 à 20 fois l'épaisseur de la couche de silicium. Ce régime se distingue tant par l'importance de la croissance latérale des grains que par l'étroitesse de la plage de densités d'énergie dans _uelle on l'observe. Cette croissance latérale semble être causée par une anisotropie du phénomène de cristallisation provoquant une extension des grains selon l'orientation privilégiée (111). De fait, l'accumulation de tirs laser provoque une augmentation de la taille des cristaux, la disparition progressive des autres orientations cristallographiques [8] et élargit la fenêtre efficace de densités d'énergie conduisant à des grains micro-métriques.

- Régime de surfusion

Au delà du régime de SLG, aucun microcristal ne subsiste et la couche de Si fondu subit une cristallisation homogène en volume menant à des grains dont le diamètre (de quelques dizaines de nm) ne dépend plus de l'énergie incidente, voire à une structure amorphe pour les films les plus fins. 


\section{PRINCIPES DE TRAITEMENT}

La mobilité des TFT étant liée à la taille des microcristaux de silicium sur lequel ils sont fabriqués, l'optimisation du traitement de cristallisation vise d'abord à accroître la taille desdits cristaux mais également à améliorer leur uniformité dont dépend celle des TFT et donc de l'écran. Cette optimisation passe par le contrôle et l'amélioration des paramètres du laser, leur adéquation avec le matériau à traiter mais également des conditions de traitement comme la température du substrat ou la nature de l'atmosphère dans laquelle est effectuée l'irradiation laser. La plupart des nombreuses techniques étudiées pour parvenir à un silicium poly-cristallin performant peuvent être classées en trois catégories selon les phénomènes physiques sur lesquels elles sont basées.

\subsection{Vitesse du front de solidification}

La relation entre la vitesse d'un front de solidification et la structure du matériau qu'il laisse derrière lui est connue de longue date mais ce n'est que récemment que l'on a montré que la taille des grains de silicium varie comme l'inverse du carré de la vitesse du front de solidification $[9,10]$. Le ralentissement de ce front a donc été recherché pour améliorer les performances des TFT. Des simulations numériques $[11,12]$ montrent que l'irradiation par une impulsion laser de $200 \mathrm{~ns}$ permet de réduire d'un facteur 2 la vitesse du front par rapport à celle résultant d'un traitement avec une impulsion de $20 \mathrm{~ns}$; ce résultat est corroboré par Mariucci [13] qui obtient des grains de silicium de $2 \mu \mathrm{m}$ de taille moyenne avec une impulsion de $160 \mathrm{~ns}$ et de $1,2 \mu \mathrm{m}$ avec une impulsion de $30 \mathrm{~ns}$ (pour une épaisseur de silicium de $40 \mathrm{~nm}$ ). L'accumulation de chaleur par conduction dans la couche de $\mathrm{SiO}_{2}$ (généralement déposée entre le substrat de verre et la couche de silicium) durant l'impulsion laser est à l'origine de ce ralentissement et de fait, le chauffage du substrat par lampe par exemple, conduit également à une diminution de la vitesse de refroidissement comme l'a démontré Fogarassy [12] (de $2,8 \mathrm{~m} / \mathrm{s}$ à $27^{\circ} \mathrm{C}$ à $1,6 \mathrm{~m} / \mathrm{s} \mathrm{à} 400^{\circ} \mathrm{C}$ pour un film de $100 \mathrm{~nm}$ et une impulsion de $200 \mathrm{~ns}$ ). L'accroissement de la durée de l'impulsion laser est donc considérée comme un moyen d'accroître les performances du silicium poly-cristallin.

Par ailleurs, la présence d'un gaz dans l'enceinte de traitement influence également la durée pendant laquelle le matériau est en fusion. Une conductivité thermique élevée du gaz environnant induit un taux de refroidissement important limitant la taille maximale des grains. Ainsi, une taille maximale de grain de $475 \mathrm{~nm}$ après un traitement conduit sous vide, n'est plus que de $235 \mathrm{~nm}$ pour un traitement effectué sous hélium [14].

\subsection{Gradient latéral de température}

La seconde catégorie de techniques étudiées pour accrôtre la taille des grains est basée sur un gradient de température imposé au film de silicium qui a pour effet d'accentuer la croissance latérale des cristaux. Lee et al.[15] ont ainsi montré que des gradients de densité d'énergie de $40 \mathrm{~mJ} / \mathrm{cm}^{2} . \mu \mathrm{m}$ et 200 $\mathrm{mJ} / \mathrm{cm}^{2} . \mu \mathrm{m}$ conduisent à des élongation de grains résultants de $0.5 \mu \mathrm{m}$ à $1.5 \mu \mathrm{m}$. On peut donc projeter sur le matériau à traiter un gradient de densité d'énergie en donnant au faisceau un profil de rampe ou utiliser un masque de phase créant périodiquement des zones 'froides' bien localisées autour desquelles de grandes structures cristallines s'étirent et peuvent atteindre des tailles de l'ordre de $7 \mu \mathrm{m}$ [16]. On peut aussi structurer certaines couches déposées sur le substrat afin d'y créer des zones ponctuelles plus ou moins absorbantes ou réfléchissantes. Ishihara [17] y parvient grâce à une matrice de plots circulaires (de 0.8 à $3.5 \mu \mathrm{m}$ de diamètre) gravés dans 1 e $\mathrm{SiO}_{2}$. L'irradiation s'effectue sur les deux faces de la structure verre/métal/SiO $\mathrm{Si}_{2}$ (gravé)/Si. Le rayonnement projeté côté verre parvient en partie jusqu'à la couche métallique qu'il échauffe. La température maximale à l'interface $\mathrm{SiO}_{2} / \mathrm{Si}$ est donc atteinte dans les creux situés entre les plots. La nucléation y est retardée ; ainsi les noyaux solides sont d'abord générés au centre des plots à partir desquels croissent jusqu'à atteindre une taille de l'ordre de 3.5 à $6.4 \mu \mathrm{m}$ selon l'épaisseur de $\mathrm{SiO}_{2}$. Dans chacune des ces techniques, la périodicités des zones chaudes et froides doit être optimisée de façon à faire se rejoindre les structures cristallines de taille maximale. Plus simplement, on peut également déposer (en couches minces) entre silicium et $\mathrm{SiO}_{2}$, des lignes métalliques qui favorisent localement le refroidissement du silicium, ou encore répartir sur la surface du silicium des îlots de $\mathrm{SiO}_{2}$ utilisé comme film anti-réfléchissant et favorisant ponctuellement le couplage optique. 


\subsection{Croissance épitaxiale latérale}

Les techniques utilisant ce phénomène reposent sur l'utilisation de cristaux de silicium comme germes donnant naissance à des structures cristallines plus importantes.

Le procédé le plus connu dit SLS (Sequential Lateral Solidification) $[18,19]$ utilise un masque consistant en une matrice de fentes en forme de chevrons de $5 \mu \mathrm{m}$ de large. Le matériau est irradié, à travers le masque que l'on déplace avec un pas de l'ordre de $0,75 \mu \mathrm{m}$ (correspondant à la croissance cristalline à chaque pas) dans le sens du chevron, après chaque irradiation. Cette technique permet la croissance d'une zone cristalline à partir de celle créé précédemment. La cristallisation perpendiculaire aux branches du chevrons résulte en la formation d'une zone centrale quasi mono-cristalline pouvant être prolongée sur plusieurs dizaines de $\mu \mathrm{m}$.

Un procédé similaire met en jeu deux étapes à deux énergies différentes. [13]. A partir d'un film de silicium amorphe et à l'aide d'un masque, on crée d'abord un matériau biphasé (bandes amorphes / polycristallines correspondant aux zones masquées et non masquées; les grains, ainsi formés, sont gros et orientés). Le matériau est alors irradié une seconde fois, sans masque. Pour une gamme d'énergie appropriée, les différences de propriétés thermodynamiques du a-Si et du poly-Si font que les zones amorphes sont totalement fondues alors que les zones poly-cristallines ne le sont que partiellement. Ces grains partiellement fondus seront les germes de la croissance épitaxiale de grains encore plus gros. Des bandes de grains de $2 \mu \mathrm{m}$ ont ainsi pu être obtenues avec des masques de 1,5 $\mu \mathrm{m}$ de large distants de 2,5 $\mu \mathrm{m}$.

\section{CONCLUSION}

Le procédé LTPS a suscité de considérables efforts de recherches mais il n'est qu'une étape de la fabrication des écrans LCD ou à OLED qui, avec l'apparition des substrats organiques, ne devra plus comporter que des procédés élémentaires de dépôt, de traitement et de structuration à basses températures.

\section{Références}

[1]J.P. Budin, Stage de formation, Club Visu SID France, 2001

[2]T. Sameshima, S. Usui, Mat. Res. Soc. Symp. Proc. 71, 435, 1986.

[3]D. Pribat, P. Legagneux, F. Plais, F. Petinot, O. Huet, C. Reita, Annales de Physique coll. C1, supp. au $\mathrm{n}^{\circ} 1 / 2$, vol. 22,1997

[4]C.V. Thompson, H.I. Smith, Appl. Phys. Lett. 44, 603, 1984

[5]H. Watanabe, H.Miki, S. Sugai. K . Kawasaki, T. Kioka, Jpn. J. Appl. Phy. Vol. 33, pp 4491-4498, 1994

[6] J.S. Im, H.J. Kim, Appl. Phys. Lett. 64, 2303, 1994

[7] J.S.Im, H.J.Kim, M.O.Thompson, Appl.Phys.Lett 63 (14),4 1993

[8]H. Kuriyama, K. Sano, S. Ishida, T. Nohda, Y. Aya, T. Kuwahara, S. Noguchi, S. Kiyama, S. Tsuda, and S. Nakano, Mat. Res. Soc. Symp. Proc. 321, 657, 1994

[9]M. Matsumura, Phys. Stat. Sol.(a), 166, pp. 715-728, 1998 .

[10]R. Ishihara et al., Electronic Letters, $31 \mathrm{n}^{\circ} 22$, pp.1956-1957, 1995.

[11]E. Fogarassy, S. de Unamuno, B. Prévot, P. Boher, M. Stehlé, D. Pribat, Appl. Phys. A 68, pp. 631$635,1999$.

[12] E.Fogarassy, S. de Unamuno, P. Legagneux, F. Plais, D. Pribat, B. Godard and M. Stehle, Thin Solid Films 337, pp. 143-147, 1999.

[13] L. Mariucci, R. Carluccio, A. Pecora, V. Foglietti, G. Fortunato et D. Della Sala, AM-LCD'99, Proc. pp. 283-286, 1999.

[14]A. Marmorstein, A.T. Voutsas, R. Solanki, J. Appl. Phys. 82 (9), 1997

[15]M. Lee, S. Moon, M. Hatano, K. Suzuki, C.Grigoropoulos., Mat. Res.Soc. Symp.Proc. 621, 2000.

[16]C.H. Oh, M. Ozawa, M. Matsumura, , Jpn. J. Appl. Phys., Part. 2 37, L492, 1998

[17]R. Ishihara, A. Burtsev, Jpn. J. Appl. Phys., 37 3B, p. 1071, 1998

[18]R. Sposili , J. Im. Appl. Phys. Lett. 69, 2864, 1996

[19] J.S. Im, R.S. Sposili et M.A. Crowder, Appl. Phys. Lett. 70, 3434, 1997 